\title{
Roderich Wahsner Die Deutsche Rechtsgeschichte und der Faschismus
}

Die Rolle der Rechtshistoriker im Faschismus ist von der rechtshistorischen Forschung der Bundesrepublik bisher fast vollkommen ausgeklammert worden. In der umfangreichen rechtshistorischen Literatur seit 1945 findet sich - soweit ersichtlich - ein einziger Beitrag, der sich mit diesem Problemkreis beschäftigt. Dabei handelt es sich um den in dieser Zeitschrift veröffentlichten, im Rahmen einer Gießener Vortragsreihe über "Nationalsozialismus und Recht" 1969 gehaltenen Vortrag von Dieter Schwab "Zum Selbstverständnis der historischen Rechtswissenschaft im Dritten Reich ". ${ }^{1}$

Die Art und Weise, in der Schwab das Thema behandelt, und das gesammelte Schweigen der anderen bundesdeutschen Rechtshistoriker zu einem ganzen Abschnitt der Geschichte ihrer Wissenschaft sind kennzeichnend für den Stand der Rechtsgeschichte in der Bundesrepublik.

II.

I. Schwabs Hauptinteresse gilt den sogenannten Germanisten, denjenigen Rechtshistorikern also, die sich seit dem 19. Jahrhundert mit der Erforschung des deutsch-germanischen Rechts befassen. Seine Untersuchung zielt von Anfang an auf folgende Fragen: Haben die germanistischen Rechtshistoriker nach 1933 versucht, ihre - aus methodischer Einsicht oder aus Resignation heraus - zunehmend »vom Anspruch auf gegenwärtige Brauchbarkeit befreite rechtsgeschichtliche Altertumswissenschaft ${ }^{2}{ }^{2}$ in den Dienst der nationalsozialistischen Rechtserneuerung aus dem Geist oder Wesen des deutschen Volkes« zu stellen? Haben sie also - entsprechend den Erwartungen zahlreicher Beiträge in der Zeitschrift des NS-Juristenbundes "Deutsches Recht ${ }^{3}$ - einen "Modellanspruch « für das alte deutsch-germanische Recht erhoben, oder haben sie unberührt von solchen dogmatischen Ansprüchen und Tagesforderungen weiterhin ihre rein historische Altertumswissenschaft betrieben?4

Schwabs Feststellungen dazu lauten, »die deutsche Rechtsgeschichte sei weder zu einer grundsätzlichen Neuorientierung ihres Selbstverständnisses gelangt“ noch habe sie "bei der 'Rechtserneuerung eine entscheidende Rolle" gespielt (S. 64); ein dogmatischer Anspruch des historischen Rechts sei von der nationalsozialistischen Jurisprudenz keineswegs akzeptiert worden (S.65); die Rechts-

\footnotetext{
1 Kritische Justiz, i 969 (Heft I), S. 58 ff.

2 A. a. O., S. 60.

3 Vgl. dazu Schwab, a. a. O., S. 58 f.

4 Diese Fragestellung zieht sich als roter Faden durch den gesamten Beitrag; vg1. a. a. O., S. 59 , $64,66,68$.
} 
geschichte habe dem Regime vielmehr nur »durch den Nachweis des genuin-germanischen Charakters einiger Parolen"wie z. B. Treue und Gefolgschaftspflicht, nützen können (S. 65). Von Schwerin, als Vertreter der Germanistik, habe zwar gefordert, daß die Rechtsgeschichte gegenwartsbezogen oder politisch betrieben werden müsse, gleichzeitig aber betont, daß auch die bloß von geschichtlicher Methode bestimmte Forschung, die rein antiquarische Forschungstätigkeit, nicht ohne Wert für die Rechtserneuerung sei (S. 67 f.). Anknüpfend hieran fährt Schwab dann fort: »Der Forscher kann nach diesem Text also wählen, ob er sich an der Suche nach dem Geist des deutsch-germanischen Rechts beteiligen und also seine Wissenschaft in den Dienst historischer Ideologieverzierung stellen oder ob er die Rationalität seiner Forschung wahren will. Das Letzte ist nun ganz überwiegend, und zwar auch bei Forschern, die wir im Banne des Nationalsozialismus wähnen dürfen, geschehen « (S. 68). Diese zentrale These enthält - und zwar in mehreren Schichten - Richtiges und Falsches zugleich.

2. Aus den Darlegungen folgt zunächst nur, daß die Germanisten - zum Teil oder auch »überwiegend « - dem deutsch-germanischen Recht keinen für die Gegenwart relevanten Modellcharakter zugesprochen und für sich selbst keinen entscheidenden Einfluß auf die nationalsozialistische Rechtserneuerung beansprucht haben.

Nicht ohne Grund mußten sich die Germanisten damals von einem durch die romanistische Schule geprägten Rechtshistoriker entgegenhalten lassen, daß statt »einer liebevollen Versenkung in die Kleinformen des genossenschaftlichen deutschen Mittelalters die große Úbersetzungsarbeit zu leisten (sei), mit der wir den gleichen Volksgeist, der diese mittelalterlichen Formen schuf, die modernen Rechtsformen finden helfen, die der Lebenswirklichkeit der deutschen Volksordnung des 20. Jahrhunderts angemessen sind «. Franz Wieacker, der dies 1935 schrieb, ${ }^{5}$ machte damit einen Einwand geltend, der von Realitätssinn zeugt. Zur "Lebenswirklichkeit der deutschen Volksordnung « gehörte die unverändert kapitalistische Form der Produktion. Die genossenschaftlichen Träumereien der Germanisten $^{\mathfrak{B}}$ waren demgegenüber an vorkapitalistischen, idyllisch-überschaubaren, fast ausschließlich auf agrarischer Produktion beruhenden gesellschaftlichen Verhältnissen orientiert. Sie enthielten damit - ähnlich wie die nationalsozialistische Ideologie selbst - eine nach rückwärts gerichtete antikapitalistische und zugleich antiliberale Komponente. Die objektive Funktion der - verwaschen genug formulierten - antikapitalistischen Punkte des Parteiprogramms der Nationalsozialisten war aber nur gewesen, breite pauperisierte und radikalisierte Bevölkerungsschichten vor allem aus dem Mittelstand an die NSDAP zu binden und gleichzeitig das spätestens seit 1932 bestehende enge Einvernehmen zwischen Hitler und den Spitzen des Industrie- und Bankkapitals sowie die Millionen-Unterstützung der Nazi-Partei durch diese $\mathrm{Kreise}^{7}$ zu verschleiern. Mit dem Sieg des Faschismus hatten diese Punkte des Programms ihre Aufgabe erfüllt; der Kapitalismus blieb unangetastet. Damit waren aber auch der Beseitigung des durch die kapitalistische Produktionsweise bedingten, funktional auf den Warentausch bezogenen, abstrakt-formalen Rechts der bürgerlichen Gesell-

\footnotetext{
5 Wandlungen der Eigentumsverfassung, S. 58.

* Die Erforschung und Darstellung des deutschen Genossenschaftsrechts war eine der Hauptinteressen Otto Gierkes (Das deutsche Genossenschaftsrecht, 4 Bde. 1867-1913; Das Wesen der menschlichen Verbände, I 902). Seit Gierke gilt das genossenschaftliche Moment geradezu als eines der entscheidenden Kennzeichen des deutsch-germanischen Rechts.

7 Vgl. dazu Czichon, Wer verhalf Hitler zur Macht? Zum Anteil der deutschen Industrie an der Zerstörung der Weimarer Republik, 2. Aufl. 1971, vor allem die im Anhang (S. s9 ff.) abgedruckten Dokumente.
} 
schaft entscheidende Grenzen gesetzt: Das Privateigentum an den Produktionsmitteln war unabdingbar; die Vertragsfreiheit konnte zwar aufs Minimale eingeschränkt werden, mußte aber im Prinzip erhalten bleiben. ${ }^{\text {a }}$ Wer unter diesen Umständen gemeint hätte, das bürgerliche Recht durch ein genossenschaftliches, deutsch-germanisches Recht ersetzen zu können, wäre ein hoffnungsloser Illusionist gewesen. Man kann daher zu Recht annehmen, daß die Germanisten in ihrer Mehrheit die Unmöglichkeit eines solchen Unterfangens erkannt haben und daß sie sich früher oder später auch der Schwierigkeiten bewußt wurden, die selbst einer Rechtserneuerung aus dem "Geiste " des deutsch-germanischen Rechts entgegenstanden.

3. Diese Einsicht bedeutet aber noch keineswegs, daß die Germanisten »überwiegend " auch "die Suche nach dem Geist des deutsch-germanischen Rechts " aufgegeben oder sich an dieser Suche »überwiegend nicht beteiligt « hätten. Die dahingehende Feststellung ist vertretbar, wenn damit lediglich gesagt werden soll, daß es auch bei den Germanisten Ausnahmeerscheinungen waren, wenn zum Problem "Rasse und Recht « beispielsweise geschrieben wurde, es sei »dank dem Führer... die gewaltige Bedeutung des Bluts und der blutsmäßigen Tradition auch für die Entwicklung des Rechts immer klarer hervorgetreten « 8 oder wenn gar - als Skurilität - die Wiedereinführung des altgermanischen Kebsverhältnisses neben der Ehe gefordert wurde. ${ }^{\theta}$

Schwabs Feststellung besagt aber mehr als das und insoweit ist sie nicht haltbar. Denn die "Suche nach dem Geist des germanisch-deutschen Rechts" war seit der Blütezeit des germanistischen $Z$ weiges der historischen Schule im 19. Jahrhundert, und ist bis auf den heutigen Tag, das erklärte Programm der deutschen Rechtsgeschichte. Dieses Programm steht noch immer im Mittelpunkt des rein antiquarischen Interesses der letzten Vertreter dieser »Altertumswissenschaft «.

"Die Aufgabe der deutschen Rechtsgeschichte ist es, die Entwicklung rechtlicher Gedanken und rechtlicher Einrichtungen im Leben des deutschen Volkes darzustellen ... Deutsche Rechtsgeschichte ... ist westgermanische Rechtsgeschichte. «10 "Unter deutscher Rechtsgeschichte verstehen wir zunächst die Rechtsgeschichte des deutschen, durch Kultur und Sprache verbundenen Volkes selbst ... Sie muß zurückgehen bis auf die Frühzeit des Germanentums und wird so zugleich zur germanischen Rechtsgeschichte. «11

Beide Bücher, denen diese Zitate entnommen sind, und von denen das zuletzt zitierte heute eine gewisse Monopolstellung besitzt, ${ }^{12}$ behandeln in ausführlicher Breite die Germanische Zeit, die Fränkische Zeit und das Mittelalter. Den rührenden, nach Sippen, Geburts- und Berufsständen wohlgeordneten Rechtsverhältnissen dieser Zeit widmen Schwerin-Thieme fast zwei Drittel, Mitteis-Lieberich sogar mehr als zwei Drittel ${ }^{13}$ des gesamten Umfangs ihrer Bücher. Beide Werke gehen ausdrücklich oder unausgesprochen davon aus, daß "stillwirkende Kräfte des Volkstums« das Recht hervorbringen und daß der sogenannte »Volks-

$7^{n} \mathrm{Vgl}$. dazu Otto Kirchbeimer, Die Rechtsordnung des Nationalsozialismus, in: KJ 197r, S. 356 (359f.).

8 Herbert Mayer, Rasse und Recht beı den Germanen und Indogermanen, I937, S. I.

$\theta$ Vgl. dazu Schwab, a. a. O., S. 59 .

10 von Schwerin-Thieme, Grundzüge der deutschen Rechtsgeschichte, 4. Aufl. 1950, S. I.

11 Mitteis-Lieberich, Deutsdhe Reditsgeschidhte, Ein Studienbud, I2. Aufl. 1970, S. 2 f. (= 10. Aufl. i 966, S. 2 f.)

12 Das belegt allein scion die Tatsache, daß dieses in der Beck'schen Reihe Juristische Kurzlehrbïcher zu einem erschwinglichen Preis angebotene Bud seit 1949 zwölf Auflagen erlebte, während das Buch yon Schwertn-Thieme nach igso nidh mehr neu aufgelegt wurde.

13 In der 10. Aufl. yon 1966 umfaßt die Behandlung von Frühzeit und Mittelalter I 86 von 242 Seıten, in der 12. Aufl. von I 97020 s von 287 Seiten. 
geist (worunter gewissermaßen naturhafte Wesenszüge eines Volkes begriffen werden) das Recht in seiner Gesamtheit entscheidend prägt. Bei Mitteis-Lieberich erscheint diese Volksgeist-Lehre in Gestalt der »im Einzel- wie im Völkerleben wirkenden Rechtsidee«, in der »Eigenart der deutschen Entwicklung«, die »nur voll verständlich wird durch den Vergleich mit anderen auf dem gleichen Kulturboden stehenden Nationen ", sowie im "germanischen Rechtsdenken ", das sich bei fast allen Völkern des europäischen Kulturkreises neben antikem und christlichem Rechtsdenken erhalten hat. ${ }^{14}$ Für Schwerin-Thieme ist das Recht »eine Seite der gesamten Kultur, hat Teil an dem Urgrund, dem Geist des Volkes «. ${ }^{15}$ Unter diesen Umständen kann es keinem Zweifel unterliegen, daß deutsche Rechtsgeschichte, soweit sie heute noch als gesonderte Disziplin betrieben wird, noch immer die "Suche nach dem Geist des deutsch-germanischen Rechts « als ihre wichtigste Aufgabe ansieht. Es sollte daher verwundern, wenn sich die Germanisten ausgerechnet im Dritten Reich an dieser Suche »überwiegend « nicht beteiligt hätten.

4. Auch die Feststellung, die Rechtshistoriker hätten ihre Wissenschaft überwiegend nicht in den Dienst »historischer Ideologieverzierung " gestellt, muß in dieser Form bezweifelt werden. Wenn mit dem Wort "Verzierung « nur die von besonderem propagandistischen Eifer geprägte historisierend-literarische Tätigkeit z. B. der Wagemann ${ }^{16}$, Meyer ${ }^{17}$ und Nicolai ${ }^{18}$ von dem vornehmeren Wirken der meisten Rechtshistoriker unterschieden werden soll, so mag die Feststellung in diesem begrenzten Sinn zutreffen. Im Kontext der vorausgegangenen Ausführungen ${ }^{19}$ muß allerdings (und soll wohl auch) der Leser assoziieren, die Rechtshistoriker hätten überwiegend nicht einmal die NS-Ideologie verziert, sie hätten dem Regime nicht einmal durch den "Nachweis des genuin-germanischen Charakters einiger Parolen« wie z. B. Treue und Gefolgschaftspflicht gedient. Richtig ist daran nur, daß von einer (nur) verzierenden Tätigkeit der Rechtshistoriker in der Tat keine Rede sein kann. Sie haben vielmehr dem Regime ganz andere und wichtigere Dienste erwiesen. Ihre objektive Funktion war es - und genau diese Funktion haben sie erfüllt -, den Nationalsozialismus als die Wiedergeburt des alten deutsch-germanischen Geistes auf dem Gebiet des Rechts zu feiern, die Machtergreifung der Nazis als Befreiung von Volk und Nation von allen Uberfremdungen auszuweisen und dem Regime auf diese Weise die Weihe der historischen Legitimation zu verleihen. Nichts anderes ist der Sinn von Ausführungen wie den folgenden:

"Die verlorengegangene Einheit von Volk und Recht durch volkstümliche, deutschem Rechtsdenken entsprechende Gestaltung unseres Rechtswesens wiederherzustellen ist das große Ziel der Rechtserneuerung, die das nationalsozialistische Dritte Reich tatkräftig in Angriff genommen hat. «

»Alles Recht ist völkisch bedingt ....

"Durch die Not und Fährlichkeiten der Gegenwart kann uns hindurch steuern nur die vom Pflichtgedanken getragene Gemeinschaftsordnung des germanisch-

14 Fußn. II, S. 2 f

15 Fußn. IO, S. 2.

${ }_{16}$ Arnold Wagemann, Deutsche Rechtsvergangenheit als Wegweiser in eine deutsche Zukunft, I 922, S. I : "Soll unser Volk zur Gesundung gelangen, so muß es wieder lernen, nach eigenem Recht zu leben... Recht ist eıne Volkserscheınung, indem es als Ergebnis einer völkischen Seelenarbeit uns vor Augen tritt, und die Anlage zum Recht... ist etwas... jedem ererbten Blut Eigentümliches

17 Vgl, oben S. 174 mit Fußn. 8 sowie ders., Recht und Volkstum, Rektoratsrede von 1929, 1933.

18 Nicolat, Rasse und Recht, 1933

10 Walter Merk, Vom Werden und Wesen des deutschen Rechts, 3. Aufl. I935, S. 9, i I u. Iog. 
deutschen Rechts, die allen werteschaffenden Volksgenossen ausreichenden Spielraum zur Entfaltung ihrer Kräfte im Dienst und im Rahmen der Volksgesamtheit gewährt. «19

"In diesem (dem NS-Staat) wird die ungermanische Zweiheit und Gegensätzlichkeit von Staat und Einzelnen durch eine Dreigliederung von Staat, Bewegung und Volk überwunden, indem die politische Bewegung den Staat wie das Volk durchdringt und zum einheitlichen Ganzen zusammenschließt. Dem entspricht die Aufnahme des germanischen auf Treue und Gefolgschaft beruhenden Führergedankens als Grundprinzip des nationalsozialistischen Staatsaufbaus. Im Führer ... schließt sich die Dreiheit zur Einheit zusammen.«20

"Die Metaphysik des germanisch-deutschen Rechts... ist das Herzstück der deutschen Rechtswissenschaft der Zukunft... die Geschichte lebt nicht nur von der Kontinuität sondern auch yon der Diskontinuität, weil sie von der Tat der Freiheit lebt, die wie ein Wunder aus der Tiefe der Seele des großen Mannes (!) bricht und der Geschichte und ihrer Not eine Wendung gibt «. ${ }^{21}$

Ob derartige Äußerungen wirklich bei der überwiegenden Anzahl der Rechtshistoriker nicht anzutreffen sind, bedürfte genauerer Prüfung. Sie vorzunehmen, ist ein hier nicht einlösbarer besonderer Forschungsauftrag für eine Rechtsgeschichte der Neuzeit. ${ }^{22}$

5. Ober die hier aufgezeigten Probleme geht $S c h w a b$ mit der Feststellung hinweg, die Rechtshistoriker hätten überwiegend die Rationalität ihrer Forschung gewahrt. Richtig mag daran sein, daß sich die rechtshistorische Literatur des Dritten Reiches im Gesamtbild nicht wesentlich von der früheren rechtshistorischen Literatur unterscheidet. Der yon $S c h w a b{ }^{23}$ erhobene Befund (der mit hoher Wahrscheinlichkeit für die Germanistik seit 1945 ganz entsprechend wäre) kann und soll nicht bezweifelt werden: "Den Bänden (der Germanistischen Abteilung der Zeitschrift für Rechtsgeschichte) ab 1933 ist - von Tagungsberichten

20 Claudius Freiberr von Schwern, Grundzüge der deutschen Rechtsgeschichte, 1934, S. 312 (= 3. Aufl. 1944, S. 36r).

21 Walter Schönfeld, Die Geschichte der Rechtswissenschaft im Spiegel der Metaphysik (= Reich und Recht in der Deutschen Philosophie, Bd. 2), 1943, S. IX f. u. 58I f.

22 Dabei verdiente die Rolle der romanistıschen Rechtshistoriker besondere Aufmerksamket. Schwab beschränkt sich insofern im wesentlichen auf die Feststellung, das gleiche wie für die Germanistik gelte »Im Ergebnıs auch für die Romanistik (S. 69). Damı soll offensichtlich gesagt werden, auch die Romanisten hätten überwiegend »die Rationalität ihrer Forschung gewahrt. Es spricht manches dafür, daß diese Aussage in bezug auf die Romanisten eine gewisse Berechtigung hat. Dann gilt aber entgegen $S c b w a b$ für sie nicht das gleiche wie für die Germanisten. Die Romanistik war nicht nur, worauf Schwab selbst hinweist, 》weithin der Gegnerschaft der nationalsozialistischen jurısprudenz ausgesetzt«, sie befand sich nicht nur win der Defensive", (S. 69) sie war vielmehr in erster Linse personell aus Deutschland verbannt, weil die Romanisten zum größten Teil jüdischer Abstammung und damut Opfer der NS-Rassegesetze waren. Daß die offensichtlich größere Distanz der Romanisten gegenüber der faschistischen Ideologie auch in ihrem Wissenschaftsverständnis begründet liegt, soll hier nur als Hypothese angedeutet werden. Die Romanıstik, die mehr dogmatische als historische Wissenschaft war, hob sich in ihrem Programm deutlich von der nationalistisch-yölkischen Position der Germanıstik ab. Die Romanisten waren weniger anfällig für das romantısch-mystizistische Volksgeıst-Denken. Sie waren zwar fixiert auf die Autorität ihrer Quelle (ursprünglich das Corpus Juris, später auch das von den Romanisten maßgeblich beeinflußte BGB); sie waren aber mit dem Versuch, den darin aufgehäuften Vorrat an abstrakt-formalen Rechtssätzen wissenschaftlich zu durchdringen und in ein logisches System einzufügen, stärker zu formaler Ratıonalität verpflichtet (vgl. zur Romanistik den Einleitungsbeıtrag »Zur Kritik der bürgerlichen Rechtsgeschichte" in diesem Heft.). Auch romanistisch geschulte Rechtshistoriker haben sich allerdings der Mitarbeit im Nationalsozialismus nicht durchweg entzogen. Franz Wicacker hat sich sogar besondere Verdienste erworben durch seine Publikation z. B. über "Wandlungen der Eigentumsverfassung «, 1935 ("Eigentum ist also die Zugehörigkeit der Sache zur Person, deren die Person zu sachgerechtem Handeln in der Volksordnung und deren die Sache zur Erfüllung ihrer Funktion in der Volksordnung bedarf « - S. 25).

23 A. a. O., S. 68 . 
abgesehen - der politische Umschwung kaum anzumerken. Art der Themenstellung, Methode und Inhalt der Aufsätze bleiben die gleichen wie in den Jahrgängen vorher. Wie auch im Großteil der monographischen Literatur werden konkrete historische Erscheinungen des Rechts untersucht, ganz überwiegend ohne den Drang zu mühsam konstruierter Nutzanwendung für die Gegenwart.« Die daraus gezogene Schlußfolgerung, die Rechtsgeschichte habe, "wo sie nicht in mehr oder minder vagen Programmatiken den deutschen Geist beschwor, ihre Rationalität « gewahrt, ${ }^{24}$ greift aber zu kurz, so lange nicht näher untersucht wird, von welcher Beschaffenheit diese "Rationalität" war, an die von der Rechtsgeschichte 1933 bruchlos angeknüpft werden konnte und angeknüptt wurde. Die Beachtung formal-rationaler Kriterien bei der Bearbeitung und Interpretation historischer Quellen kann wohl nicht mit Rationalität schlechthin gleichgesetzt werden. Die Rationalität des wissenschaftlich-methodischen Verfahrens ist kein ausreichender Schutz gegen die Irrationalität der vermittelten Inhalte. Sie hat jedenfalls die deutsche Rechtsgeschichte auch und gerade als reine Altertumswissenschaft nicht davor bewahrt, Wasserträgerdienste für den Nationalsozialismus zu verrichten. Eine Wissenschaft, die das aus Sitte, Gewohnheit und Religion sich allmählich herauslösende Recht einer frühen, in Verhältnissen der Ungleichheit, der abgestuften Unfreiheit und der unmittelbaren Gewalt, rein für die Bedarfsdeckung produzierenden Gesellschaftsformation (die einen Warenverkehr nicht oder nur in schwach entwickelter Form kannte) aus rein antiquarischem Interesse untersucht, erhebt unausgesprochen dieses Recht oder den Geist dieses Rechts zum Vorbild für die Gestaltung oder zum Maßstab für die Bewertung des Rechts der Gegenwart, solange sie nicht die spezifische Differenz jenes alten deutschen Rechts zum abstrakten, mit dem Anspruch auf Allgemeinheit auftretenden Formalrecht der auf kapitalistischer Produktion beruhenden, warentauschenden bürgerlichen Gesellschaft als Problem erkennt und zum eigentlichen Gegenstand ihres Erkenntnisinteresses erhebt.

Eine Wissenschaft, die demgegenüber - nach eigenem Zeugnis - sich erstmals 1947 (wenn auch nur vorübergehend) selbst »zum Problem wurde und die bis dahin " ihr Werk mit anscheinend nachtwandlerischer Sicherheit verrichtete ${ }^{25}$; eine Wissenschaft, deren Publikationen der "politische Umschwung (von 1933) kaum anzumerken « ist, bei der "Art der Themenstellung, Methode und Inhalt der Aufsätze« und der "monographischen Literatur " in dieser Zeit die gleichen bleiben wie vorher ${ }^{26}$; eine Wissenschaft schließlich, die erst durch die Zeit nach x 945 zur "Neubewertung « ihres "geistigen Besitzes gezwungen « und mit dem Gebot konfrontiert wurde, »sich vor sich selbst und der Allgemeinheit zu rechtfertigen ${ }^{27}$ - eine solche historische Wissenschaft war entweder in Themenstellung, Methode und Inhalt vollkommen ungefährlich für den Nationalsozialismus oder aber aufs engste mit der faschistischen Ideologie verwandt.

Die Tatsache, daß sich noch in der germanistisch-rechtshistorischen Literatur der Bundesrepublik faschistische Inhalte ausmachen lassen, ist ein Indiz für die geistige Verwandtschaft. Einige weitere Beispiele aus den bereits zitierten Lehrbüchern zur deutschen Rechtsgeschichte sollen das belegen.

Nach einigen Hinweisen auf die Bismarck'sche Sozialgesetzgebung und auf die gesetzlichen Bestimmungen über den Arbeitsschutz aus der Zeit vor I9I4 wird bei Schwerin-Thieme $\mathrm{e}^{28}$ festgestellt:

24 A. a. O., S. 69.

25 Vgl. Heinrich Mitteis, Vom Lebenswert der Rechtsgeschichte, 1947, S. 7.

26 Schwab, a. a. O., S. 68.

27 Heinrich Mittess (Fußn. 25), S. 8.

28 (Fußn. IO), S. 247. 
»Doch beruhte diese Gesetzgebung ebenso wie das Arbeitsrecht der Weimarer Republik und seit 1945 nach wie vor auf dem Gegensatz von Arbeitgebern und Arbeitnehmern. Der Versuch einer Uberbrückung, wie ihn das nationalsozialistische Arbeitsrecht gemacht hat, verdient aber in ruhigeren Zeiten wieder erwogen zu werden «.

Hier wird das auf Führerprinzip und Gefolgschaftstreue und auf dem Verbot von Streik und Gewerkschaften beruhende faschistische Arbeitsrecht offen als vorbildlich empfohlen. Genau wie in der faschistischen Ideologie wird der Widerspruch von Lohnarbeit und Kapital geleugnet. Der für ruhigere Zeiten wieder empfohlene Versuch einer Uberbrückung basiert auf der Annahme einer vorgegebenen, den Gegensatz in sich aufhebenden Gemeinschaft, als welche von der germanistischen Rechtsgeschichte noch heute die Volksgemeinschaft begriffen wird. Diese Volksgemeinschaft wird auch im Schlußwort von MitteisLieberich sichtbar, das - später völlig umformuliert - noch in der ro. Auflage von $1966^{29}$ wie folgt lautete: "Es ist die große Aufgabe unserer Zeit..., das Volk wieder an den Staat heranzuführen, ihm Liebe und Vertrauen zu seinem Recht wiederzugeben und den Staat so zu gestalten, daß die schöpferischen Kräfte des Volkstums in ihrer Mannigfaltigkeit bewahrt und zu einträchtigem Zusammenwirken im Inneren und mit anderen Völkern gebracht werden können. Erst wenn Volk, Staat, Recht und Gerechtigkeit wieder eins sind, dann wird sich der Ring der deutschen Rechtsgeschichte geschlossen haben."

Wo von den »schöpferischen Krä̈ten des Volkstums « die Rede ist, da werden die "zersetzenden Kräfte unausgesprochen mitgedacht. Gesellschaftliche Widersprüche, die in gesellschaftlichen Konflikten und Kämpfen ausgetragen werden, existieren nicht. Das Ubel der Zeit ist die Entfremdung des Volkes vom Staat und die fehlende Einheit von Volk, Staat (weiland noch Führer)( Recht und Gerechtigkeit. Das typisch faschistische Heilmittel zur solchermaßen begriffenen Trennung von Staat und bürgerlicher Gesellschaft sind "Heranführen an den Staat ", "Liebe und Vertrauen zum Recht « und eine Gestaltung des Staates in der Weise, daß die "schöpferischen « also nicht die zersetzenden Kräfte des Volkstums zu »einträchtigem Zusammenwirken gebracht werden können «.

Wie sehr auch im einzelnen faschistische Positionen nachwirken, zeigen Wertungen von Mitteis-Lieberich in Bezug auf die neuere deutsche Geschichte. Das von Bismarck mit "Blut und Eisen « geschaffene Kaiserreich entstand »als selbstgenügsamer deutscher Nationalstaat « ${ }^{30}$. Dieses selbstgenügsame Reich wurde I9I4 - so als hätte die kaiserlich-deutsche Regierung nicht nach einer Weltmachtstellung gestrebt, als hätte es keine Kriegsvorbereitungen, keine Kriegshetze und keine Annexionspläne des Alldeutschen Verbandes und des Verbunds von Militär und Großindustrie gegeben ${ }^{31}$ - »des äußeren und bald auch des inneren Friedens beraubt. Der Weltkrieg überfiel das deutsche Verfassungsleben unvorbereitet « ${ }^{32}$. Nicht der Krieg und die militärische Niederlage, das Kriegsgewinnlertum der Kapitalisten, die Unterdrückung und die Ausbeutung der Arbeiter, der Hunger und das wachsende Elend der arbeitenden Massen ${ }^{33}$ - sondern: "Die Novemberrevolution von 1918, Folge des militärischen Zusammenbruchs und

20 (Fußn. I I), S. 242.

30 (Fußn. I1), Io. Aufl. i966, S. 23 I.

31 Vgl. dazu Arthur Rosenberg, Entstehung der Weimarer Republik, I 96I, S. 35 ff. $(64,67,95$ ff.) und Fritz Fischer, Der Griff nach der Weltmacht, 3. Aufl. 1964 sowie ders., Weltmacht oder Niedergang, Deutschland im ersten Weltkrieg, 2. Aufl. 1968.

32 (Fußn. I I), io. Aufl. 1966, S. 233.

33 Vgl. dazu vor allem Rosenberg (Fußn. 31), S. I 17 ff., I60 f., 181 ff. 
einer allgemeinen Führungslosigkeit, stürzte Deutschland in ein Chaos « ${ }^{34} \mathrm{Mit}$

dieser Feststellung wird die Novemberrevolution nicht als Ausdruck des Klassenkampfes gewertet, der sich im Laufe des Krieges aufs äußerste zugespitzt hatte, sondern als ein Problem von Führungslosigkeit. Damit wird einerseits die Illusion genährt, starke Männer, wie Hindenburg und Ludendorf hätten die Revolution verhindern können, andererseits wird zugleich die schon in den zwanziger Jahren von den Faschisten und von der gesamten politischen Reaktion verbreitete Dolchstoßlegende kolportiert, wonach der militärische Sieg nur durch einen von zersetzenden Kräften in der Heimat geführten Dolchstoß in den Rücken dieser starken Männer vereitelt wurde.

6) Interessanterweise stellt auch Schwabs5 fest, daß es "Übereinstimmungen zwischen germanistischer Tradition und nationalsozialistischer Schlagwortideologie gegeben habe. Als Beispiele werden u. a. erwähnt die These von der Überfremdung der deutschen Kultur durch die Geltung des römischen Rechts, die alte germanistische Forderung nach Volkstümlichkeit und Volksverbundenheit des Rechts, die Feindschaft der Germanisten gegen das abstrakte Formalrecht des BGB und der Gedanken von der sozialen Natur und der Gemeinschaftsbindung subjektiver Rechte. Das eigentliche Interesse gilt aber nicht dieser ideologischen Übereinstimmung im einzelnen. Sie wird als mehr oder weniger zufällig begriffen und nicht als das eigentliche Problem erkannt. Nirgends wird die Frage aufgeworfen, was die germanistischen Rechtshistoriker - und zwar schon lange vor 1933 - mit ihren typischen Denkmustern und Denkstrukturen zur Entwicklung der faschistischen Ideologie und zu ihrer Verbreitung in der Sozialisation der Juristen beigetragen haben. Auch diese Frage umreißt ein ganzes Programm für eine deutsche Rechtsgeschichte des 19. und 20. Jahrhunderts.

Der Erkenntnis dieser Seite des Problems von Nationalsozialismus und Rechtsgeschichte setzt bereits der Titel des Vortrags eine Grenze. Es ist zwar wichtig, das "Selbstverständnis der historischen Rechtswissenschaft im Dritten Reich « zu kennen und dieses Selbstverständnis sah offensichtlich nicht ganz so aus, wie der Vortrag es zu vermitteln sucht. Es genügt aber nicht zu wissen, welche Vorstellungen sich die Rechtshistoriker in dieser Zeit von sich selbst und ihrem gesellschaftlichen Auftrag gemacht haben. Diese Kenntnis allein besagt nämlich nichts über die wirkliche Geschichte, über die objektive Funktion, die die Germanisten für den Faschismus erfüllt haben. Insoweit gilt für die Rechtsgeschichte entsprechend, was Marx und Engels in der "Deutschen Ideologie "36 über die deutsche Geschichtswissenschaft ihrer Zeit feststellten: "Während im gewöhnlichen Leben der Shopkeeper sehr wohl zwischen dem zu unterscheiden weiß, was Jemand zu sein vorgibt, und dem, was er wirklich ist, so ist unsere Geschichtsschreibung noch nicht zu dieser trivialen Erkenntnis gekommen. Sie glaubt jeder Epoche aufs Wort, was sie von sich selbst sagt und sich einbildet."

III.

Ein Schlaglicht auf die Situation der Rechtsgeschichte in der Bundesrepublik wirft die Tatsache, daß Schwabs Beitrag der bisher einzige eines bundesdeutschen Rechtshistorikers ist, der das Verhältnis von Rechtsgeschichte und Nationalso-

34 (Fußn, I r), 10. Aufl. r966, S. 234.

35 A. a. O., S. 62 f.

36 MEW Bd. 3, S. 49. 
zialismus überhaupt einer Untersuchung für wert befunden hat. Die älteren, selbst noch betroffenen Rechtshistoriker bewältigen ihre eigene Vergangenheit auf ihre Weise. Hans Thieme z. B. gab 1949 das Lehrbuch zur deutschen Rechtsgeschichte von Schwerins in neuer Auflage heraus, strich dabei u. a. die oben (Fußn. 20) zitierte Passage und ersetzte sie durch ein fades Lamento über den "totalitären Charakter « des Dritten Reiches und über die "von seiner Gefolgschaft systematisierte Willkür (!) eines einzigen Mannes «37. Walter Schönfeld ${ }^{38}$ entdeckte nun, daß es sich beim Nationalsozialismus um eine "Gewaltherrschaft « gehandelt habe, begriff deren Sturz als "ungeheuerliche Katastrophe«, besann sich auf die christlichen Werte des Abendlandes und gab "nach längerem Schweigen « sein früheres Buch unter neuem Titel und weitgehend umgearbeitet in zweiter Auflage heraus. Seine "Grundlegung der Rechtswissenschaft " unterscheidet sich von dem früheren Werk wesentlich dadurch, daß - an Stelle des Nationalsozialismus - als neu "gelegter Grund « des Rechts und der Rechtsgeschichte entdeckt wird: "Jesus Christus « ${ }^{39}$, als unsere und unseres Rechts alleinige Rettung verheißen wird: "Das Evangelium « ${ }^{40}$ Franz Wieacker ${ }^{41}$ geht zwar in kürzeren Abschnitten seiner "Privatgeschichte der Neuzeit « auf die Zivilrechtswissenschaft im Dritten Reich, auf die nationalsozialistische Zivilrechtsgesetzgebung und auf die damals geübte Kritik am Zivilrechtssystem, am Allgemeinen Teil des BGB und an der Abstraktheit des bürgerlichen Rechts ein; die Geschichte der Rechtsgeschichte im Dritten Reich wird aber geflissentlich vorenthalten. Während Wieacker die Lage nach 1945 als "Krise des deutschen Nationalstaatsgedankens" oder als eine der "politischen Katastrophen « begreift, ${ }^{42}$ wird für Heinz Lieberich, den Bearbeiter des Mitteis'schen Kurzlehrbuchs zur deutschen Rechtsgeschichte, das Jahr I 945 schlicht und einfach zum "Jahre Null «.43

Auch dieses Kapitel deutscher Rechtsgeschichte bedarf einer gründlichen Untersuchung. Schon diese kurze Skizze rechtfertigt die Hypothese, daß die Rechtshistoriker der Bundesrepublik, insbesondere die letzten noch lebenden Vertreter des Faches $>$ Deutsche Rechtsgeschichte der Tradition ihrer Wissenschaft über die Niederlage des Faschismus hinaus treu geblieben sind.

Es ist die Tradition einer - scheinbar - rein antiquarischen Wissenschaft, die das für die Gegenwart relevante Erkenntnisinteresse von Rechtsgeschichte für eine allgemeine Theorie des Rechts, für die Frage von Genesis und Geltung des Rechts, für seine Bedingtheit und Funktionalität auf der Grundlage des ökonomischen Lebensprozesses der Menschen ebenso ausblendet wie die Realität der jeweiligen historischen Gesellschaftsformation, insbesondere die Realität der bürgerlich-kapitalistischen Gesellschaft. Es ist die Tradition einer "geschichtlichen "Wissenschaft, die in dieser doppelten, für das bürgerliche Denken typischen ahistorichen Ausblendung längst vergangene Epochen der Geschichte glorifiziert und auf diese Weise - scheinbar >unpolitisch - politisch zur Gegenwart Stellung nimmt.

Die biologisch-organizistische Volksgeistlehre der Germanisten; ihre Vorliebe für die idyllischen, überschaubaren, nach Geburts- und Berufsständen wohlgeordneten, rein oder überwiegend auf agrarischer Produktion beruhenden Verhält-

37 von Schwersn-Thieme (Fußn. 10), S. 335.

38 Grundlegung der Rechtswissenschaf, 195x (2. völlig umgearbeitete Auflage des Buches von

1943 - Fußn. 21), Vorwort sowie S. 3 u. 4.

3n (Fußn. 38), S. I, I 3 u. passim.

40 (Fußn. 38), S. 539.

41 Privatrechtsgeschichte der Neuze1t, 2. Aufl. 1967, S. s14 f. (mit Fußn. 2), s53 ff., s56 f.

42 (Fußn. 41), S. 19 (in Fußn. I 8 ) und S. 25.

43 (Fußn. II), 12. Aufl. 1970, S. 248 . 
nisse der Frühzeit und des Mittelalters; ihre Versenkung in die genossenschaftlichen Kleinformen des germanisch-deutschen Rechts (Wieacker); der damit vollzogene, antikapitalistisch getönte Rückzug aus der Gegenwart ${ }^{44}$; ihr nationalistisch-deutschtümelnder Kampf gegen die Ergebnisse der als Überfremdung begriffenen Rezeption des römischen Rechts; die aus dem germanisch-deutschen Genossenschaftsrecht entwickelte Gemeinschaftsideologie, insbesondere die Ideologie der Volksgemeinschaft (Einheit von »Volk, Staat, Recht und Gerechtigkeit «); die damit verbundene Leugnung der Existenz gesellschaftlicher Klassen und Klassenkonflikte; die antidemokratische Konzeption einer zu Treue und Gehorsam gegenüber charismatischen Führern verpflichteten, ständisch gegliederten Gefolgschaft; die willfährige Verbreitung von Legenden zur neueren deutschen Geschichte: in all diesen Punkten, die sich in den Lehrbüchern der Germanisten zum Teil noch heute nachweisen lassen, entsprach die Deutsche Rechtsgeschichte genau der Art von »historischer « Wissenschaft, die der Nationalsozialismus brauchte. Der Faschismus hätte daher die deutsche Rechtsgeschichte erfinden müssen, wären nicht die deutschen Rechtshistoriker »Miterfinder « wesentlicher Versatzstücke der faschistischen Ideologie gewesen, hätten sie nicht schon lange vor I 933 faschistisches Denken in den Sozialisationsprozeß der Juristen hineingetragen und in ihrem aufgehäuften Wissensstoff die historischen Legitimationsmuster für die faschistische Herrschaft bereitgehalten.

44 Vgl. u. a. Karl Siegfried Bader, Aufgaben und Methoden des Rechtshistorikers, i95I, S. 8 ("Der unbeirte Vertreter einer vom Tagesgeschehen unberührten Geisteswissenschaft zu sein, das ist das Nächste und Wichtigste, was man dem Rechtshistoriker wünschen kann. «) 